\title{
On Bessel Functions Related with Certain Classes of Analytic Functions with respect to Symmetrical Points
}

\author{
Afis Saliu (D), ${ }^{1}$ Khalida Inayat Noor, ${ }^{1}$ Saqib Hussain $\left(\mathbb{D},{ }^{2}\right.$ and Maslina Darus $\mathbb{D}^{3}$ \\ ${ }^{1}$ Department of Mathematics, COMSATS University Islamabad, Park Road, Tarlai Kalan, Islamabad 45550, Pakistan \\ ${ }^{2}$ Department of Mathematics, COMSATS University Islamabad, Abbottabad Campus, Abbottabad 22060, Pakistan \\ ${ }^{3}$ Department of Mathematical Sciences, Universiti Kebangsaan Malaysia, Bangi, 43600, Selangor, Malaysia
}

Correspondence should be addressed to Afis Saliu; saliugsu@gmail.com

Received 5 November 2020; Revised 27 December 2020; Accepted 18 January 2021; Published 9 February 2021

Academic Editor: Ming-Sheng Liu

Copyright (C) 2021 Afis Saliu et al. This is an open access article distributed under the Creative Commons Attribution License, which permits unrestricted use, distribution, and reproduction in any medium, provided the original work is properly cited.

In the present investigation, subclasses of analytic functions with respect to symmetrical points which are defined by the generalized Bessel functions of the first kind of order $\mu$ are introduced. Furthermore, some alluring geometric properties of these classes, which include inclusion property, integral-preserving properties, coefficients, and distortion results are studied. Moreover, some consequences of our results are also given.

\section{Introduction}

Geometric function theory (GFT) is the area of complex analysis which deals with the geometric characterization of analytic functions, established around the turn of the twentieth century[1]. It is a known fact that the study of special functions plays a significant role in GFT. One reason is that solutions of extremal problems can be frequently written in terms of special function. Another reason is that some important conformal mappings are given by special function. For example, the conformal mapping of an annulus onto the complement of two closed segments on the real axis and the conformal mapping of a square onto a rectangle are expressed by elliptic functions (see [2]). In recent times, the solution of Bieberbach conjecture by de Branges is obtained with the help of special functions [3].

Bessel function is one of the most significant special functions. It is therefore important for solving many problems in engineering, physics, and mathematics (see $[4,5])$. For instance, it is used for velocity and stress derivation in the rotational flow of Burge's fluid flowing through an unbounded round channel [6].

In recent times, many researchers paid their attention on establishing various conditions under which a Bessel function has some certain geometric properties such as close-to-convexity (univalency), starlikeness, and convexity in frame of a unit disc $U$ (see [7-11]).

The objective of this manuscript is twofold. Firstly, Bessel functions of the first kind of order $\mu$ is used to introduce new generalized starlike and convex functions with respect to symmetrical points, which was first initiated and studied by Sakaguchi [12] and Das and Sign [13]. Moreover, we examine some interesting geometric properties of these classes, which include inclusion property, integral-preserving properties, coefficients, and distortion results.

\section{Materials and Methods}

Now, we give some basic preliminaries and definitions that play the integral part in obtaining our main results.

Consider $b, c, \mu \in \mathbb{C}$ (the set of complex numbers) and the second-order linear homogenous differential equation

$$
z^{2} w^{\prime \prime}(z)+b z w^{\prime}(z)+\left[c z^{2}-\mu^{2}+(1-b) \mu\right] w(z)=0,
$$

which is a natural extension of Bessel's equation. The solution $w(z)$ (see [14]) of (1) has a series representation:

$$
w(z)=\sum_{n=0}^{\infty} \frac{(-c)^{n}}{n ! \Gamma(\mu+n+(b+1 / 2))}\left(\frac{z}{2}\right)^{2 n+\mu}, \quad z \in \mathbb{C} .
$$


Differential equation (2) allows the investigation of Bessel function of the first kind of order $\mu[15,16]$ (the case $b=c=1$ ), modified Bessel function $[15,16]$ (the case $b=1$, $c=-1$ ), and the spherical Bessel Function [16] (the case $b=2$, $c=1)$. Using the well-known Pochhammer symbol $(a)_{n}$ with $a \neq 0,-1,-2, \ldots$, we consider the function $\varphi_{\mu, b, c}(z)$ defined by the transformation

$$
\begin{aligned}
\varphi_{\mu, b, c}(z) & =2^{\mu} \Gamma\left(\mu+\frac{b+1}{2}\right) z^{1-\mu} w(\sqrt{z}) \\
& =z+\sum_{n=2}^{\infty} \frac{(-c)^{n-1}}{4^{n-1}(\mu+(b+1 / 2))_{n-1}} a_{n} z^{n}, \\
& \text { where } \mu+\frac{b+1}{2} \neq 0,-1,-2, \ldots
\end{aligned}
$$

Let $\mathscr{A}$ denote the class of normalized analytic functions $f(z)$ in $U$ given by the representation

$$
f(z)=z+\sum_{n=2}^{\infty} a_{n} z^{n}
$$

Then, the convolution of $f(z)$ and $g(z)=z+\sum_{n=2}^{\infty} b_{n} z^{n}$ denoted by $f(z) * g(z)$ is defined by

$$
f(z) * g(z)=z+\sum_{n=2}^{\infty} a_{n} b_{n} z^{n},
$$

and we say $f(z)$ is subordinate to $g(z)$ (written as $f(z) \prec g(z))$ if there exists a Schwarz function $w(z)$ such that $f(z)=g(w(z)), z \in U$.

Let $\mathscr{B}_{\mu, b}^{c}: \mathscr{A} \longrightarrow \mathscr{A}$ be an operator defined by

$$
\begin{aligned}
\mathscr{B}_{\mu, b}^{c} f(z) & =\varphi_{\mu, b, c}(z) * f(z) \\
& =\sum_{n=1}^{\infty} A_{n} z^{n},
\end{aligned}
$$

$$
\text { where } \begin{aligned}
A_{n} & =\frac{(-c)^{n-1}}{4^{n-1}(\mu+(b+1 / 2))_{n-1}(n-1) !} a_{n}, \\
a_{1} & =1 .
\end{aligned}
$$

From (6), we have the identity relation

$$
z\left(\mathscr{B}_{\mu+1, b}^{c} f(z)\right)^{\prime}=(\mu+p) \mathscr{B}_{\mu, b}^{c} f(z)-(\mu+p-1) \mathscr{B}_{\mu+1, b}^{c} f(z),
$$

where $p=(b-1 / 2)$. It is easy to observe from (7) that

$$
z\left(\Phi_{\mu+1, b}^{c} f(z)\right)^{\prime}=(\mu+p) \Phi_{\mu, b}^{c} f(z)-(\mu+p-1) \Phi_{\mu+1, b}^{c} f(z)
$$

where

$$
\Phi_{\mu, b}^{c} f(z)=\frac{1}{2}\left[\mathscr{B}_{\mu, b}^{c} f(z)-\mathscr{B}_{\mu, b}^{c} f(-z)\right]
$$

Let $\phi(z)$ be a convex univalent function in $U$ with $\phi(0)=$ 1 and $\operatorname{Re} \phi(z)>0$ in $U$. Ma and Minda and Kim examined the classes $C(\phi), S^{*}(\phi)$ (see [17]), and $K(\phi)$ (see [18]) using the subordination techniques. In particular, for $-1 \leq B<$ $A \leq 1, \quad C[(1+A z / 1+B z)] \equiv C[A, B], \quad S^{*}[(1+A z / 1+B z)] \equiv$ $S^{*}[A, B][19]$ and $K[(1+A z / 1+B z)] \equiv K[A, B]$ [20] and $S^{*}[(1+z / 1-z)] \equiv S^{*}$ and $C[(1+z / 1-z)] \equiv C[21]$.

Definition 1. Let $f \in A$. Then, $f \in S_{\mu, b, c}^{s}(\phi)$ if and only if

$$
\frac{z\left(\mathscr{B}_{\mu, b}^{c} f(z)\right)^{\prime}}{\Phi_{\mu, b}^{c} f(z)} \prec \phi(z), \quad z \in U,
$$

and $f \in C_{\mu, b, c}^{s}(\phi)$ if and only if

$$
\frac{\left(z\left(\mathscr{B}_{\mu, b}^{c} f(z)\right)^{\prime}\right)^{\prime}}{\left(\Phi_{\mu, b}^{c} f(z)\right)^{\prime}}<\phi(z), \quad z \in U .
$$

We note that $f \in C_{\mu, b, c}^{s}(\phi) \Longleftrightarrow z f^{\prime} \in S_{\mu, b, c}^{s}(\phi)$. If $\phi(z)=$ $(1+A z / 1+B z),-1 \leq B<A \leq 1$, we set $S_{\mu, b, c}^{s}(1+A z / 1+$ $B z)=S_{\mu, b, c}^{s}[A, B]$ and $C_{\mu, b, c}^{s}(1+A z / 1+B z)=C_{\mu, b, c}^{s}[A, B]$. It is worthy of note that if $\left((-c)^{n-1} / 4^{n-1}(\mu+(b+1 / 2))_{n-1}(n-\right.$ $1) !)=1$ in (6) and $\phi(z)=(1+z / 1-z)$, then the classes $S_{\mu, b, c}^{s}(\phi)$ and $C_{\mu, b, c}(\phi)$ reduce to the classes $S_{s}^{*}$ and $C_{s}$, consisting of functions which are starlike and convex with respect to symmetrical points $[12,13,22-24]$.

The following lemmas are the key tools to prove our main results.

Lemma 1 (see $[19,25])$. If $p(z)<(1+A z / 1+B z)$, $-1 \leq B<A \leq 1$, then

$$
\frac{1}{2 \pi} \int_{0}^{2 \pi}|p(z)|^{2} \mathrm{~d} \theta \leq \begin{cases}1+\frac{(B-A)^{2}}{B^{2}}\left(\frac{B^{2} r^{2}}{1-B^{2} r^{2}}\right), & B \neq 0, \\ 1+A^{2} r^{2}, & B=0,\end{cases}
$$

$$
\frac{1-A r}{1-B r} \leq|p(z)| \leq \frac{1+A r}{1+B r} .
$$

Lemma 2 (see [26]). Let $g(z)$ be an univalent function in $U$. Then, there exists $z_{1}$ with $\left|z_{1}\right|=r$ such that for all $z \in U$,

$$
\left|z-z_{1}\right||g(z)| \leq \frac{2 r^{2}}{1-r^{2}} .
$$

Lemma 3 (see [21]). Let $\psi(z)$ be convex in $U$ with $\psi(0)=1$. Suppose also that $\lambda(z)$ is analytic in $U$ with $\operatorname{Re} \lambda(z) \geq 0(z \in U)$. If $p(z)$ is analytic in $U$ with $p(0)=1$, then

$$
p(z)+\lambda(z) z p^{\prime}(z) \prec \psi(z) \text { in } U,
$$

which implies that

$$
p(z) \prec \psi(z) \text { in } U \text {. }
$$


Lemma 4 (see $[21,27])$. Let $\psi(z)$ be convex in $U$ with $\operatorname{Re}(\beta \psi(z)+\gamma)>0$. If $p(z)$ is analytic in $U$ with $p(0)=\psi(0)$, then

$$
p(z)+\frac{z p^{\prime}(z)}{\beta p(z)+\gamma} \prec \psi(z) \Rightarrow p(z) \prec \psi(z) \text { in } U .
$$

\section{Results and Discussion}

Theorem 1. If $f \in S_{\mu, b, c}^{s}(\phi)$, then $\Phi_{\mu, b}^{c} f(z) \in S^{*}(\phi)$.

Proof. Let $f \in S_{\mu, b, c}^{s}(\phi)$. Then,

$$
\frac{z\left(\mathscr{B}_{\mu, b}^{c} f(z)\right)^{\prime}}{\Phi_{\mu, b}^{c} f(z)}=p(z), \quad p(z)<\phi(z) .
$$

Replacing $z$ with $-z$ in (18) and using the fact that $\Phi_{\mu, b}^{c} f(z)$ is an odd function, we have

$$
\frac{z\left(\mathscr{B}_{\mu, b}^{c} f(-z)\right)^{\prime}}{\Phi_{\mu, b}^{c} f(z)}=p(-z), \quad p(-z)<\phi(z),
$$

which combined with (18) gives

$$
\frac{z\left(\Phi_{\mu, b}^{c} f(z)\right)^{\prime}}{\Phi_{\mu, b}^{c} f(z)}=p(z), \quad p(z)+p(-z) .
$$

By subordination property, we have that $\Phi_{\mu, b}^{c} f(z) \in S^{*}(\phi)$.

Corollary 1. The function $\mathscr{B}_{\mu, b}^{c} f(z)$ belongs to $K(\phi)$ and hence is univalent in $U$.

Setting $\quad\left((-c)^{n-1} / 4^{n-1}(\mu+(b+1 / 2))_{n-1}(n-1) !\right)=1$ and choosing $\phi(z)=(1+z / 1-z)$ in Theorem 1 , we are led to the result of Sakaguchi [12] contained in the following corollary.

Corollary 2. Every function $f(z)$ in $S_{s}^{*}$ is a close-to-convex function.

Theorem 2. Suppose $\operatorname{Re}(\mu+(b+1 / 2))>1$ and $\Phi_{\mu, b}^{c} f(z) \epsilon$ $S^{*}(\phi)$. Then,

$$
S_{\mu, b, c}^{s}(\phi) \subset S_{\mu+1, b, c}^{\mathcal{S}}(\phi)
$$

Proof. Consider

$$
\frac{z\left(\mathscr{B}_{\mu, b}^{c} f(z)\right)^{\prime}}{\Phi_{\mu, b}^{c} f(z)}=h(z) .
$$

From relation (7), (22) can be written as

$$
(\mu+p) \mathscr{B}_{\mu, b}^{c} f(z)=h(z) \Phi_{\mu+1, b}^{c} f(z)+(\mu+p-1) \mathscr{B}_{\mu+1, b}^{c} f(z) .
$$

Differentiating (23) and applying (8), we obtain

$$
\begin{aligned}
(\mu+p) z\left(\mathscr{B}_{\mu, b}^{c} f(z)\right)^{\prime} & =z h^{\prime}(z) \Phi_{\mu+1, b}^{c} f(z) \\
& +(\mu+p) h(z) \Phi_{\mu, b}^{c} f(z),
\end{aligned}
$$

i.e.,

$$
(\mu+p) \frac{z\left(\mathscr{B}_{\mu, b}^{c} f(z)\right)^{\prime}}{\Phi_{\mu, b}^{c} f(z)}=(\mu+p) h(z)+z h^{\prime}(z) \frac{\Phi_{\mu+1, b}^{c} f(z)}{\Phi_{\mu, b}^{c} f(z)} .
$$

It follows from (8) that

$$
\frac{\Phi_{\mu+1, b}^{c} f(z)}{\Phi_{\mu, b}^{c} f(z)}=\frac{\mu+p}{H(z)+\mu+p-1},
$$

where

$$
H(z)=\frac{z\left(\Phi_{\mu+1, b}^{c} f(z)\right)^{\prime}}{\Phi_{\mu+1, b}^{c} f(z)} \prec \phi(z) .
$$

In view of (25) and (26), we obtain

$$
\frac{z\left(\mathscr{B}_{\mu, b}^{c} f(z)\right)^{\prime}}{\mathscr{B}_{\mu, b}^{c} f(z)}=h(z)+\frac{z h^{\prime}(z)}{H(z)+\mu+p-1} .
$$

Since $\operatorname{Re}(\mu+(b+1 / 2))>1$ and $\Phi_{\mu+1, b}^{c} f(z) \in S^{*}(\phi)$, then $\operatorname{Re}(H(z)+\mu+p-1)>0$. Hence, by Lemma 3, $h(z) \prec \phi(z)$, i.e., $f \in S_{\mu, b, c}^{s}(\phi)$. This completes the proof.

Corollary 3. Let $\operatorname{Re}(\mu+(b+1 / 2))>1$ and $\Phi_{\mu+1, b}^{c} f(z) \epsilon$ $S^{*}[A, B]$. Then,

$$
S_{\mu, b, c}^{\mathcal{S}}[A, B] \subset S_{\mu+1, b, c}^{\mathcal{s}}[A, B] .
$$

Corollary 4. Suppose that all the conditions of Theorem 2 are satisfied. Then,

$$
C_{\mu, b, c}^{s}(\phi) \subset C_{\mu+1, b, c}^{s}(\phi)
$$

Proof By Theorem 2. we have that

$$
\begin{aligned}
f \in C_{\mu, b, c}^{s}(\phi) & \Longleftrightarrow z f^{\prime} \in S_{\mu, b, c}^{s}(\phi) \Longleftrightarrow z f^{\prime} \in S_{\mu+1, b, c}^{s}(\phi) \\
& \Longleftrightarrow f \in C_{\mu+1, b, c}^{s}(\phi) .
\end{aligned}
$$

Theorem 3. Let $J_{\alpha}(f(z))$ be defined by the integral transformation

$$
J_{\alpha}(f(z))=\frac{\alpha+1}{2 z^{\alpha}} \int_{0}^{z} t^{\alpha-1}(f(t)-f(-t)) \mathrm{d} t, \quad \alpha=1,2,3, \ldots
$$

and suppose $f \in S_{\mu, b, c}^{s}(\phi)$. Then, $\mathscr{B}_{\mu, b}^{c} J_{\alpha}(f(z)) \in S^{*}(\phi)$.

Proof. Let

$$
\frac{z\left(\mathscr{B}_{\mu, b}^{c} J_{\alpha}(f(z))\right)^{\prime}}{\Phi_{\mu, b}^{c} J_{\alpha}(f(z))}=p(z),
$$

where $p(z)$ is analytic in $U$ with $p(0)=1$. From (32) and applying the operator $\mathscr{B}_{\mu, b}^{c}$, we obtain 


$$
z\left(\mathscr{B}_{\mu, b}^{c} J_{\alpha}(f(z))\right)^{\prime}-\alpha \mathscr{B}_{\mu, b}^{c} J_{\alpha}(f(z))=(\alpha+1) \Phi_{\mu, b}^{c} f(z),
$$

i.e.,

$$
\mathscr{B}_{\mu, b}^{c} J_{\alpha}(f(z))[p(z)+\alpha]=(\alpha+1) \Phi_{\mu, b}^{c} f(z) .
$$

Differentiating (35) logarithmically, we obtain

$$
p(z)+\frac{z p^{\prime}(z)}{p(z)+\alpha}=\frac{z\left(\Phi_{\mu, b}^{c} f(z)\right)^{\prime}}{\Phi_{\mu, b}^{c} f(z)} .
$$

and in view of Theorem 1 , it follows that

$$
p(z)+\frac{z p^{\prime}(z)}{p(z)+\alpha}<\phi(z),
$$

which by Lemma 4 implies $p(z)<\phi(z)$. Thus, $\mathscr{B}_{\mu, b}^{c} J_{\alpha}(f(z)) \in S^{*}(\phi)$.

Corollary 5. Let $f \in C_{\mu, c, b}^{s}(\phi)$. Then, $\mathscr{B}_{\mu, b}^{c} J_{\alpha}(f(z)) \in C(\phi)$.

Proof.

$$
\begin{aligned}
f \in C_{\mu, c, b}^{s}(\phi) & \Longleftrightarrow z f^{\prime}(z) f \in S_{\mu, c, b}^{s}(\phi) \\
& \Longleftrightarrow \mathscr{B}_{\mu, b}^{c} J\left(z f^{\prime}(z)\right) \in S^{*}(\phi) \text { (by Theorem 3) } \\
& \Longleftrightarrow z\left(\mathscr{B}_{\mu, b}^{c} J_{\alpha}(f(z))\right)^{\prime} \in S^{*}(\phi) \\
& \Longleftrightarrow \mathscr{B}_{\mu, b}^{c} J_{\alpha}(f(z)) \in C(\phi) .
\end{aligned}
$$

Corollary 6. Let $-1 \leq B<A \leq 1$ and $f \in S_{\mu, c, b}^{s}[A, B]$. Then, $\mathscr{B}_{\mu, b}^{c} J_{\alpha}(f(z)) \in S^{*}[A, B]$. Similarly, if $f \in C_{\mu, c, b}^{s}[A, B]$, then $\mathscr{B}_{\mu, b}^{c} J_{\alpha}(f(z)) \in C^{*}[A, B]$.

Setting $\quad\left((-c)^{n-1} / 4^{n-1}(\mu+(b+1 / 2))_{n-1}(n-1) !\right)=1$ and choosing $\phi(z)=(1+z / 1-z)$ in Theorem 3 , we are led to the results of Das and Sign [13] contained in the following corollaries.

Corollary 7. Let $f \in S_{s}^{*}$. Then, $J_{0}(f(z)) \in S^{*}$.

Corollary 8. Let $f \in C_{s}$. Then, $J_{0}(f(z)) \in C$.

Theorem 4. If $f \in S_{\mu, c, b}^{s}[A, B]$, then for $c \neq 0$,

$$
\left|a_{n}\right| \leq\left\{\begin{array}{l}
\frac{D_{1}(A, B) 4^{n-1}(\mu+(b+1 / 2))_{n-1}(n-1) ! n^{(-A / 2 B)}}{|c|^{n-1}}, \quad B \neq 0, \\
\frac{D_{1}(A) 4^{n-1}(\mu+(b+1 / 2))_{n-1}(n-1) !}{n|c|^{n-1}}, \quad B=0 .
\end{array},\right.
$$

where $D_{1}(A, B)$ is a constant that depends on $A$ and $B$, while $D_{1}(A)$ only depends on $A$.

Proof. Since $f \in S_{\mu, c, b}^{s}[A, B]$, then by Cauchy Theorem,

$$
\left|n A_{n}\right| \leq \frac{1}{2 \pi r^{n}} \int_{0}^{2 \pi}\left|\Phi_{\mu, b}^{c} f(z)\right||p(z)| \mathrm{d} \theta,
$$

where $A_{n}$ is given by (6) and $p(z) \prec(1+A z / 1+B z), z \in U$. From Theorem 1, we knew that for $\phi(z)=(1+A z / 1+B z)$, the function $\Phi_{\mu, b}^{c} f(z)$ is an odd starlike function of Janowski type. Thus,

$$
\Phi_{\mu, b}^{c} f(z) \prec \begin{cases}\frac{z}{\left(1+B z^{2}\right)^{(B-A) / 2 B}}, & B \neq 0, \\ z \exp \left(\frac{A z^{2}}{2}\right), & B=0 .\end{cases}
$$

By Cauchy-Schwarz inequality, subordination property, and Lemma 1 for the case $B \neq 0$, (40) implies

$$
\begin{aligned}
\left|n A_{n}\right| & \leq \frac{1}{r^{n}}\left(\frac{1}{2 \pi} \int_{0}^{2 \pi}\left|\Phi_{\mu, b}^{c} f(z)\right|^{2} \mathrm{~d} \theta\right)^{1 / 2}\left(\frac{1}{2 \pi} \int_{0}^{2 \pi}|p(z)|^{2} \mathrm{~d} \theta\right)^{1 / 2}, z=r e^{i \theta} \\
& \leq \frac{1}{r^{n}}\left(\frac{1}{2 \pi} \int_{0}^{2 \pi} \frac{r^{2}}{\left(1+B r^{2}\right)^{(A-B) / 2 B}} \mathrm{~d} \theta\right)^{1 / 2}\left(1+\frac{(B-A)^{2} r^{2}}{1-B^{2} r^{2}}\right)^{1 / 2} \\
& =\frac{r}{r^{n}\left(1+B r^{2}\right)^{(A-B) / 2 B}}\left(1+\frac{(B-A)^{2} r^{2}}{1-B^{2} r^{2}}\right)^{1 / 2} .
\end{aligned}
$$

Observe that since $-1 \leq B<1$, we have

$$
\begin{aligned}
& \frac{1}{1+B r^{2}} \leq \frac{1}{1-r^{2}}, \\
& \frac{1}{1-B r^{2}}<\frac{1}{1-r^{2}} .
\end{aligned}
$$

Thus,

$$
\left|n A_{n}\right| \leq \frac{\sqrt{1+(B-A)^{2}}}{r^{n}(1-r)^{(B-A) / 2 B+(1 / 2)}} .
$$

Let $r=1-1 / n(n \longrightarrow \infty)$; we obtain from (6) and (44) that 


$$
\left|a_{n}\right| \leq \frac{D_{1}(A, B) 4^{n-1}(\mu+(b+1 / 2))_{n-1}(n-1) ! n^{(-A / 2 B)}}{|c|^{n-1}},
$$

where $D_{1}(A, B)$ is given in Theorem 4 .

For the case $B=0$, we implement Lemma 1 and subordination property in (41) and follow the procedures for the case $B \neq 0$ to obtain

$$
\left|n A_{n}\right| \leq \exp \left(\frac{A}{2}+1\right) \sqrt{1+A^{2}},
$$

which completes the proof by using (6).

Remark 1. If we allow $\left((-c)^{n-1} / 4^{n-1}(\mu+(b+1 / 2))_{n-1}\right)=1$ and choose $A=1, B=-1$ in Theorem 3 , it follows that

$$
\left|a_{n}\right|=O(1) n^{(1 / 2)}, \quad \text { where } O(1) \text { is } a \text { constant. }
$$

Since $S_{s}^{*}$ is a subclass of the class of close-to-convex function (see [12]), it shows that our index of $n$ is a nice one.

Theorem 5. Let $f \in S_{\mu, b, c}^{s}[A, B]$. Then,

$$
\left\|a_{n+1}|-| a_{n}\right\| \mid \leq \begin{cases}\frac{D_{2}(A, B) 4^{n-1}(\mu+((b+1) / 2))_{n-1}(n-1) ! n}{|c|^{n-1}}, & B \neq 0, \\ \frac{D_{2}(A) 4^{n-1}(\mu+((b+1) / 2))_{n-1}(n-1) !}{|c|^{n-1}}, & B=0,\end{cases}
$$

where $D_{2}(A, B)$ is a constant that depends on $A$ and $B$, while $D_{2}(A)$ only depends on $A$.
Proof. Let $z_{1}$ be a complex number with $\left|z_{1}\right|=r$. Then, by Cauchy Theorem, we obtain

$$
\begin{aligned}
\left|z_{1}(n+1) A_{n+1}-n A_{n}\right| & \leq \frac{1}{2 \pi r^{n+1}} \int_{0}^{2 \pi}\left|z_{1}-z\right|\left|z\left(\mathscr{B}_{\mu, b}^{c} f(z)\right)^{\prime}\right| \mathrm{d} \theta \\
& =\frac{1}{2 \pi r^{n+1}} \int_{0}^{2 \pi}\left|\left(z_{1}-z\right) \Phi_{\mu, b}^{c} f(z)\right||p(z)| \mathrm{d} \theta,
\end{aligned}
$$

where $p(z)<(1+A z / 1+B z),-1 \leq B<A \leq 1$. Following the techniques used in Theorem 4 and using Lemma 2, we obtain

$$
\left|z_{1}(n+1) A_{n+1}-n A_{n}\right| \leq \frac{2}{r^{n-1}(1-r)}\left(\frac{1+(B-A)^{2}}{1-r}\right)^{1 / 2} .
$$

Taking $r=1-1 / n(n \longrightarrow \infty)$ and choosing $z_{1}=((n$ $\left.(-c / 4)^{n-1} /(\mu+(b+1 / 2))_{n-1}(n-1) !\right) /\left((n+1)(-c / 4)^{n} /(\mu+\right.$ $\left.\left.(b+1 / 2))_{n}(n) !\right)\right)$, we have the result. The case $B=0$ also follows the same procedures.

Theorem 6. If $f \in S_{\mu, b, c}^{s}[A, B]$, then

$$
\frac{\left[2 n-\left(1-(-1)^{n}\right)\right]^{2}|c|^{2}\left|a_{n}\right|^{2}}{4^{n-1}(\mu+(b+1 / 2))_{n-1}(n-1) !} \leq \sum_{m=1}^{n-1} \begin{cases}\frac{\left[A\left(1-(-1)^{m}\right)-2 B m\right]^{2}|c|^{2}\left|a_{m}\right|^{2}}{4^{m-1}(\mu+(b+1 / 2))_{m-1}(m-1) !}, & B \neq 0 \\ \frac{4 A^{2}|c|^{2}\left|a_{m}\right|^{2}}{4^{m-1}(\mu+(b+1 / 2))_{m-1}(m-1) !}, & B=0 .\end{cases}
$$

Proof. Let $f \in S_{\mu, b, c}^{s}[A, B]$. Then, there exists an analytic function $w(z)=\sum_{n=1}^{\infty} w_{n} z^{n} \quad$ with $\quad w(0)=0$ and $|w(z)|<1(z \in U)$ such that which is equivalent to

$$
z\left(\mathscr{B}_{\mu, b}^{c} f(z)\right)^{\prime}-\Phi_{\mu, b}^{c} f(z)=\left[A \Phi_{\mu, b}^{c} f(z)-B z\left(\mathscr{B}_{\mu, b}^{c} f(z)\right)^{\prime}\right] w(z) .
$$

Thus, 


$$
\begin{aligned}
& \sum_{n=1}^{\infty}\left[2 n-\left(1-(-1)^{n}\right)\right] A_{n} z^{n} \\
& \quad=\left\{\sum_{n=1}^{\infty}\left[A\left(1-(-1)^{n}\right)-2 B n\right] A_{n} z^{n}\right\} w(z),
\end{aligned}
$$

where we have used (6), (9), and (53). Comparing the coefficients of $z^{n}$ in (54), we obtain

$$
\begin{aligned}
& 2(A-B) A_{1} w_{n-1}-2 B A_{2} w_{2}+\cdots \\
& \quad-\left[A\left(1-(-1)^{n}\right)-2 B(n-1)\right] A_{n-1} w_{1}=\left[2 n-\left(1-(-1)^{n}\right)\right] A_{n} .
\end{aligned}
$$

The coefficients combination on the right side of (55) depends only upon the coefficients combination $2(A-B) A_{1}$, $-2 B A_{2}, 2(A-3 B) A_{3}, \ldots,\left[A\left(1-(-1)^{n}\right)-2 B(n-1)\right] A_{n-1}$ of the left side. Therefore, we can write $(54)$ as

$$
\begin{aligned}
w(z) & \sum_{m=1}^{n-1}\left[A\left(1-(-1)^{m}\right)-2 B m\right] A_{m} z^{m} \\
\quad= & \sum_{m=1}^{n}\left[2 m-\left(1-(-1)^{m}\right)\right] A_{m} z^{m}+\sum_{m=n+1}^{\infty} c_{m} z^{m},
\end{aligned}
$$

for some $c_{m}, n+1 \leq m<\infty$. Squaring the moduli of both sides of (56), integrating around the circle $|z|=r$, and using Parseval's theorem, we note that

$$
\begin{gathered}
\sum_{m=1}^{n}\left[2 m-\left(1-(-1)^{m}\right)\right]^{2}\left|A_{m}\right|^{2} r^{2 m}+\sum_{m=n+1}^{\infty}\left|c_{m}\right|^{2} r^{2 m} \\
<\sum_{m=1}^{n-1}\left[A\left(1-(-1)^{m}\right)-2 B m\right]^{2}\left|A_{m}\right|^{2} r^{2 m} .
\end{gathered}
$$

Therefore,

$$
\begin{aligned}
& \sum_{m=1}^{n}\left[2 m-\left(1-(-1)^{m}\right)\right]^{2}\left|A_{m}\right|^{2} r^{2 m} \\
& \quad \leq \sum_{m=1}^{n-1}\left[A\left(1-(-1)^{m}\right)-2 B m\right]^{2}\left|A_{m}\right|^{2} r^{2 m} .
\end{aligned}
$$

Taking limit as $r \longrightarrow 1^{-}$, we obtain the required result. In the following theorem, $\operatorname{erf}(\mathrm{x})$ for an arbitrary $x$ denotes the error function and we need an Euler integral representation for the special class of hypergeometric functions given in [28] and defined as follows.

For $\operatorname{Re}(c)>\operatorname{Re}(b)>0$,

$$
\begin{aligned}
{ }_{3} F_{2}\left(a, \frac{b}{2}, \frac{b+1}{2} ; \frac{c}{2}, \frac{c+1}{2} ; x\right) \\
\quad=\frac{\Gamma(c)}{\Gamma(b) \Gamma(c-b)} \int_{0}^{1} t^{b-1}(1-t)^{c-b-1}\left(1-x t^{2}\right)^{-a} \mathrm{~d} t .
\end{aligned}
$$

Theorem 7. Let $f \in S_{\mu, b, c}^{s}[A, B]$. Then, for $|z|=r(0<r<1)$,

$$
\wedge(A, B, r) \leq\left|\mathscr{B}_{\mu, b}^{c} f(z)\right| \leq \vee(A, B, r)
$$

where

$\vee(A, B, r)=$

$$
\left\{\begin{array}{l}
\frac{r}{B}\left[A_{3} F_{2}\left(\frac{B-A}{2 B}, \frac{1}{2}, 1 ; 1, \frac{3}{2} ;-B r^{2}\right)+(B-A) \sum_{k=0}^{\infty} \frac{\left(-B r^{2}\right)^{k}}{k+1}{ }_{3} F_{2}\left(\frac{B-A}{2 B}, \frac{k+1}{2}, \frac{k+2}{2} ; \frac{k+2}{2}, \frac{k+3}{2} ;-B r^{2}\right)\right], \quad B \neq 0, \\
\sqrt{\frac{-\pi}{2 A}} \operatorname{erf}\left(\sqrt{\frac{-A}{2} r}\right)+\left[\exp \left(\frac{A r^{2}}{2}\right)-1\right], \quad B=0, A<0,
\end{array}\right.
$$

$\wedge(A, B, r)=$

$$
\left\{\begin{array}{l}
\frac{r}{B}\left[F_{2}\left(\frac{B-A}{2 B}, \frac{1}{2}, 1 ; 1, \frac{3}{2} ; B r^{2}\right)+(B-A) \sum_{k=0}^{\infty} \frac{\left(B r^{2}\right)^{k}}{k+1}{ }_{3} F_{2}\left(\frac{B-A}{2 B}, \frac{k+1}{2}, \frac{k+2}{2} ; \frac{k+2}{2}, \frac{k+3}{2} ; B r^{2}\right)\right] B \neq 0, \\
\sqrt{\frac{\pi}{2 A}} \operatorname{erf}\left(\sqrt{\frac{A}{2} r}\right)+\left[\exp \left(\frac{-A r^{2}}{2}\right)-1\right], \quad B=0, A>0 .
\end{array}\right.
$$


Equality is obtained for the function

$$
\mathscr{B}_{\mu, b}^{c} f(z)=\int_{0}^{z}\left(1+B y v^{2}\right)^{-(A-B) / 2 B} \frac{1+A y v}{1+B y v} \mathrm{~d} v,
$$

if $z=r(0<r<1)$ and $y= \pm 1$.

Proof. Since $f \in S_{\mu, b, c}^{s}[A, B]$, then

$$
\left(\mathscr{B}_{\mu, b}^{c} f(z)\right)^{\prime}=\Phi_{\mu, b}^{c} f(z) p(z),
$$

where $\Phi_{\mu, b}^{c} f(z) \in S^{*}[A, B]$ by Theorem 1 and $p \prec(1+A z / 1+B z)$. Using (41) and subordination property, we have that

$$
\begin{aligned}
\left|\left(\mathscr{B}_{\mu, b}^{c} f(z)\right)\right| & \leq \int_{0}^{r} \frac{\left|\Phi_{\mu, b}^{c} f\left(\rho e^{i \theta}\right)\right|\left|p\left(\rho e^{i \theta}\right)\right|}{\rho} \mathrm{d} \rho \\
& \leq \int_{0}^{r}\left(1+B \rho^{2}\right)^{-(B-A) / 2 B} \frac{1+A \rho}{1+B \rho} \mathrm{d} \rho \\
& =\frac{A}{B} \int_{0}^{r}\left(1+B \rho^{2}\right)^{-(B-A) / 2 B} \mathrm{~d} \rho+\frac{B-A}{B} \sum_{k=0}^{\infty}(-B)^{k} \int_{0}^{r} \rho^{k}\left(1+B \rho^{2}\right)^{-(B-A) / 2 B} \\
& =\frac{A r}{B} \int_{0}^{1}\left(1+\left(B r^{2}\right) u^{2}\right)^{-(B-A) / 2 B} \mathrm{~d} u+\frac{(B-A) r}{B} \sum_{k=0}^{\infty}(-B r)^{k} \int_{0}^{r} u^{k}\left(1+\left(B r^{2}\right) u^{2}\right)^{-(B-A) / 2 B} \mathrm{~d} u .
\end{aligned}
$$

By adopting (13) and (64), we obtain

Applying hypergeometric function (59), we obtain the upper bound for the case $B \neq 0$. In case $B=0$, applying (13) and using (64) in (66), we obtain

$$
\begin{aligned}
\left|\left(\mathscr{B}_{\mu, b}^{c} f(z)\right)\right| & \leq \int_{0}^{r} \exp \left(\frac{A \rho^{2}}{2}\right) \mathrm{d} \rho+\int_{0}^{r} A \rho \exp \left(\frac{A \rho^{2}}{2}\right) \mathrm{d} \rho \\
& =\sqrt{\frac{-2}{A}} \int_{0}^{\sqrt{(-A / 2)} r} \exp \left(-u^{2}\right) \mathrm{d} u+\left[\exp \left(\frac{A r^{2}}{2}\right)-1\right], \frac{A \rho^{2}}{2}=-u^{2}, A<0 \\
& =\sqrt{\frac{-\pi}{2 A}} \operatorname{erf}\left(\sqrt{\frac{-A}{2} r}\right)+\left[\exp \left(\frac{A r^{2}}{2}\right)-1\right] .
\end{aligned}
$$

This establishes the upper bound. To prove the lower bound, we consider a point $z_{0}\left(\left|z_{0}\right|=r<1\right)$ such that $\left|\left(\mathscr{B}_{\mu, b}^{c} f(z)\right)\right| \geq\left|\left(\mathscr{B}_{\mu, b}^{c} f\left(z_{0}\right)\right)\right|(\forall z:|z|=r)$. Let $C$ be an arc in $U$ which is mapped by the function $w=\left(\mathscr{B}_{\mu, b}^{c} f(z)\right)$ onto a line segment $L$ connecting origin to the point $\left(\mathscr{B}_{\mu, b}^{c} f\left(z_{0}\right)\right)$ and lying completely in the image of $U$ under $\left(\mathscr{B}_{\mu, b}^{c} f(z)\right)$. Thus, by (13) and (65), we obtain

$$
\begin{aligned}
\left|\mathscr{B}_{\mu, b}^{c} f(z)\right| & \geq\left|\mathscr{B}_{\mu, b}^{c} f\left(z_{0}\right)\right|=\int L|\mathrm{~d} w| \\
& =\int C\left|\left(\mathscr{B}_{\mu, b}^{c} f(z)\right)^{\prime}\right||\mathrm{d} z| \\
& \geq \int_{0}^{r}\left(1-B \rho^{2}\right)^{-(B-A) / 2 B} \frac{1-A \rho}{1-B \rho} \mathrm{d} \rho .
\end{aligned}
$$


Adopting similar procedures as used in finding the upper bounds from (65), we obtain the desired result.

For $A=1$ and $B=-1$, we obtain a more reduced form of Theorem 7 which is contained in the following corollary.

Corollary 9. If $f \in S_{\mu, b, c}^{s}[1,-1]$, then

$$
\ln \left(\frac{1+r}{\sqrt{1+r^{2}}}\right) \leq\left|\mathscr{B}_{\mu, b}^{c} f(z)\right| \leq \frac{r}{1-r}(r<1) .
$$

This bound cannot be improved.

Corollary 10. If $f \in S_{\mu, b, c}^{s}[0,-1]$, then

$$
\begin{aligned}
\ln \left(\frac{1+r}{\sqrt{1+r^{2}}}\right)^{(1 / 2)} \leq & \left|\mathscr{B}_{\mu, b}^{c} f(z)\right| \leq \ln \left(\frac{1+r}{1-r}\right)^{(1 / 4)} \\
& +\frac{r}{2(1-r)}(r<1) .
\end{aligned}
$$

This bound is sharp.

Remark 2. In respect of the lower bounds of $\left|\mathscr{B}_{\mu, b}^{c} f(z)\right|$ for the classes $S_{\mu, b, c}^{s}[1,-1]$ and $S_{\mu, b, c}^{s}[0,-1]$, given by $(69)$ and (70), respectively, we note that the disc of the maximum radius is contained in the image domain $\mathscr{B}_{\mu, b}^{c} f\left(U_{r}\right)$ if $f \in S_{\mu, b, c}^{s}[1,-1]$ and $f \in S_{\mu, b, c}^{s}[0,-1]$, respectively, where $U_{r}\{z \in \mathbb{C}:|z|=r<1\}$.

In view of Remark 2, we note that as $r \longrightarrow 1^{-}$in the lower bound of both Corollaries 9 and 10, we have the following results giving the omission values for the classes $S_{\mu, b, c}^{s}[1,-1]$ and $S_{\mu, b, c}^{s}[0,-1]$.

Corollary 11. Let $f \in S_{\mu, b, c}^{s}[1,-1]$ and $w \in \mathbb{C}$ be such that $f(z) \neq w(z \in U)$. Then, $|w|>0.34657359$.

Corollary 12. Let $f \in S_{\mu, b, c}^{s}[0,-1]$ and $w \in \mathbb{C}$ be such that $f(z) \neq w(z \in U)$. Then, $|w|>0.173286795$.

\section{Conclusion}

Bessel functions are essential in many branches of mathematics and applied mathematics. Recently, there has been a clear interest on Bessel and hypergeometric functions from the point of view of geometric function theory. As a result, we presented some subclasses of analytic functions with respect to symmetrical points, which were associated with Bessel function. The geometric properties of these aforementioned classes which include integral-preserving properties, coefficients, and distortion results were studied. As a consequence of our investigation, some relevant special cases were pointed out. In addition, to capture more new results under the current examination, new idea and applications can be investigated with some positive and novel outcomes in various fields of science, especially in mathematics. These new investigations will be presented in future research work being processed by the authors of the present article.

\section{Data Availability}

No data were used to support the findings of the study.

\section{Conflicts of Interest}

The authors declare that they have no conflicts of interest.

\section{Acknowledgments}

The authors would like to thank the Rector of COMSATS University Islamabad, Islamabad, Pakistan, for providing excellent research and academic environment. The fourth author is supported by UKM grant (GUP-2019-032).

\section{References}

[1] M. Darus, "A note on geometric function theory and recent studies," in AIP Conference Proceedings, vol. 1522, pp. 29-32, 2013.

[2] S.-L. Qiu and M. Vuorinen, "Special functions in geometric function theory," Handbook of Complex Analysis, vol. 2, pp. 621-659, 2005.

[3] L. V. Ahlfors, "Classical Analysis: Present and Future," in Proceedings of the Symposium. On the occasion of the solution of the Bieberbach Conjecture. Mathematical Surveys Monographs 21, A. H. Baerntein, D. Drasin, P. L. Duren, and A. Marden, Eds., pp. 1-6, American Mathematical Society, Providence, RI, USA, 1986.

[4] L. Durand, "Complex asymptotics in $\lambda$ for the gegenbauer functions $\mathrm{C} \lambda \alpha(\mathrm{z})$ and $\mathrm{D} \lambda \alpha(\mathrm{z})$ with $\mathrm{z} \in(-1,1)$," Symmetry, vol. 11, no. 12, p. 1465, 2019.

[5] C. Fang, G. He, and S. Xiang, "Hermite-type collocation methods to solve volterra integral equations with highly oscillatory Bessel kernels," Symmetry, vol. 11, no. 2, p. 168, 2019.

[6] M. Imran, D. L. C. Ching, R. Safdar, I. Khan, M. Imran, and K. Nisar, "The solutions of non-integer order burgers' fluid flowing through a round channel with semi analytical technique," Symmetry, vol. 11, no. 8, p. 962, 2019.

[7] A. Baricz, "Geometric properties of generalized Bessel functions," Publicationes Mathematicae Debrecen, vol. 73, no. 1-2, pp. 155-178, 2008.

[8] A. Baricz, "Geometric properties of generalized Bessel functions," in Generalized Bessel Functions of the First Kind, pp. 23-69, Springer, Berlin, Heidelberg, 2010.

[9] Á. Baricz and S. Ponnusamy, "Starlikeness and convexity of generalized Bessel functions," Integral Transforms and Special Functions, vol. 21, no. 9, pp. 641-653, 2010.

[10] K. S. Nisar, D. L. Suthar, S. D. Purohit, and H. Amsalu, "Unified integrals involving product of multivariable polynomials and generalized Bessel functions," The Bulletin of Parana's Mathematical Society, vol. 38, no. 6, pp. 73-83, 2020.

[11] D. L. Suthar and A. Pradhan, "Generalized fractional integral operators of certain class of starlike and convex functions," Vijnana Parishad Anusandhan Patrika, vol. 51, no. 3, pp. 253-261, 2008.

[12] K. Sakaguchi, "On a certain univalent mapping," Journal of the Mathematical Society of Japan, vol. 11, no. 1, pp. 72-75, 1959.

[13] R. N. Das and P. Singh, "On subclasses of schlicht mapping," Indian Journal of Pure and Applied Mathematics (IJPAM), vol. 8, no. 8, pp. 864-872, 1977.

[14] S. Mahmood, N. Raza, E. S. A. AbuJarad, G. Srivastava, H. M. Srivastava, and S. N. Malik, "Geometric properties of 
certain classes of analytic functions associated with a q-integral operator," Symmetry, vol. 11, no. 5, p. 719, 2019.

[15] A. Baricz, "Generalized Bessel functions of the first kind," Lecture note in Mathematics, Vol. 1994, Springer-Verlag, Berlin, Germany, 2010.

[16] G. N. Watson, A Treatise on the Theory of Bessel Function, Cambridge University Press, Cambridge, EN, UK, 2nd edition, 1995.

[17] W. Ma and D. Minda, "A unified treatment of some special classes of univalent functions," in Proceedings of the International Conference on Complex Analysis at the Nankai Institute of Mathematics, pp. 157-169, Tianjin, China, January 1992.

[18] Y. C. Kim, J. H. Choi, and T. Sugawa, "Coefficient bounds and convolution properties for certain classes of close-to-convex functions," Proceedings of the Japan Academy, Series A, Mathematical Sciences, vol. 76, no. 6, pp. 95-98, 2000.

[19] W. Janowski, "Some extremal problems for certain families of analytic functions I," Annales Polonici Mathematici, vol. 28, no. 3, pp. 297-326, 1973.

[20] B. S. Mehrok, "A subclass of close-to-convex functions," International Journal of Mathematical Analysis, vol. 4, pp. 1319-1327, 2010.

[21] S. S. Miller and P. T. Mocanu, "Differential subordinations, theory and applications," Series of Monographs and Textbooks in Pure and Applied Mathematics, Vol. 225, Marcel Dekker, New York/Basel, NY, USA, 2000.

[22] F. Ghanim and M. Darus, "On new subclass of analytic functions with respect to symmetric points," Archivum Mathematicum, vol. 45, no. 3, pp. 179-188, 2009.

[23] W. R. Ibrahim and M. Darus, "New symmetric differential and integral operators defined in the complex domain," Symmetry, vol. 11, p. 7, 2019.

[24] H. M. Srivastava, N. Khan, M. Darus, S. Khan, Q. Z. Ahmad, and S. Hussain, "Fekete-szegö type problems and their applications for a subclass of $q$-starlike functions with respect to symmetrical points," Mathematics, vol. 8, p. 5842, 2020.

[25] N. E. Cho, V. Kumar, and V. Ravichandran, "Arc length for the Janowski classes," Analele Stiintifice ale Universitatii Al I Cuza din Iasi-Matematica, Tomul LXV, vol. 1, , 2019.

[26] G. Golusin, "On distortion theorems and coefficients of univalent functions," Matematicheskii Sbornik, vol. 19, pp. 183-203, 1946.

[27] R. M. El-Ashwash, M. K. Aouf, and M. Darus, "Differential subordination results for analytic functions," Asian-European Journal of Mathematics, vol. 06, no. 04, Article ID 1350044, 2013.

[28] K. A. Driver and S. J. Jhonston, "An integral representation of some hypergeometric functions," Electronic Transactions on Numerical Analysis, vol. 25, pp. 115-120, 2006. 